Conclusion Specialist MDT discussion influenced changes in diagnosis in $44.2 \%$ of patients. The majority of IPF cases discussed are external referrals. This has implications for design and delivery of specialist ILD services.

Case selection for Pirfenidone does not appear based on lung function differences. This has implications for guidelines for its use.

\section{P30 EFFICACY OF PULSED CYCLOPHOSPHAMIDE AND METHYL-PREDNISOLONE THERAPY IN PATIENTS WITH PROGRESSIVE INTERSTITIAL LUNG DISEASE}

${ }^{1} \mathrm{P}$ Dutta, ${ }^{2} \mathrm{P}$ Avery, ${ }^{1} \mathrm{~L}$ Mansell, ${ }^{3} \mathrm{~B}$ Griffiths, ${ }^{3} \mathrm{I}$ Forrest, ${ }^{1} \mathrm{AJ}$ Simpson. 'Institute of Cellular Medicine, Newcastle University, Newcastle Upon Tyne, UK; ${ }^{2}$ School of Mathematics \& Statistics, Newcastle University, Newcastle Upon Tyne, UK; ${ }^{3}$ The Newcastle Upon Tyne Hospitals NHS Foundation Trust, Newcastle Upon Tyne, UK

\subsection{6/thoraxjn-2015-207770.167}

Introduction Therapeutic options for progressive interstitial lung disease (ILD) are limited, as no pharmacological intervention has been shown to improve mortality significantly. Intravenous (IV) pulsed cyclophosphamide and methyl-prednisolone are administered in some centres for rapidly progressive ILD, based on small clinical improvements in patients with ILD secondary to systemic sclerosis. We studied the outcome of patients with ILD who received IV cyclophosphamide and methyl-prednisolone therapy in our centre.

Methods Patients were identified from a database of those receiving cyclophosphamide. We reviewed the case notes of patients receiving IV cyclophosphamide between January 2010 to August 2014, comparing the rate of change in forced vital capacity (FVC) and transfer factor for carbon monoxide (TLco) before and after therapy. Adverse events were also recorded.

Results Records from 53 patients with a mean age of 60 years (range 39 - 81 years) were reviewed; 29 (55\%) were male. Diagnosis included Connective Tissue Related-ILD (21), Idiopathic NSIP (12), Chronic Hypersensitivity Pneumonitis (8), IPF (6) and Undifferentiated-ILD (6). The median number of cyclophosphamide pulses received was 6 (range 1 to 23). After completion of cyclophosphamide, 41 (77\%) patients received follow on immunosuppressive therapy in the form of mycophenolate mofetil (37 patients), azathioprine or rituximab.

The average rate of change of lung function was significantly less after cyclophosphamide therapy both for FVC (Figure 1) $\mathrm{p}=0.0004$ and TLco $\mathrm{p}=0.00015$.

Whilst on therapy $8(15 \%)$ patients had bone marrow suppression (new onset neutrophil count $<2.0 \times 10^{9} / 1$ and/or platelet count $\left.<140 \times 10^{9} / 1\right), 4(7 \%)$ had elevated liver function tests, $3(6 \%)$ had abnormal renal function, and one was investigated for microscopic haematuria. 32 episodes of infections were documented of which 21 were of respiratory origin. 25 (71\%) out of 35 patients who suffered from an adverse event were able to complete therapy.

Conclusion In our single centre, retrospective study, pulsed intravenous cyclophosphamide and methyl-prednisolone was associated with stabilisation of lung function in a mixed cohort of patients with progressive ILD. Adverse events were common but transient and managed with dose reduction and/or delayed schedule.

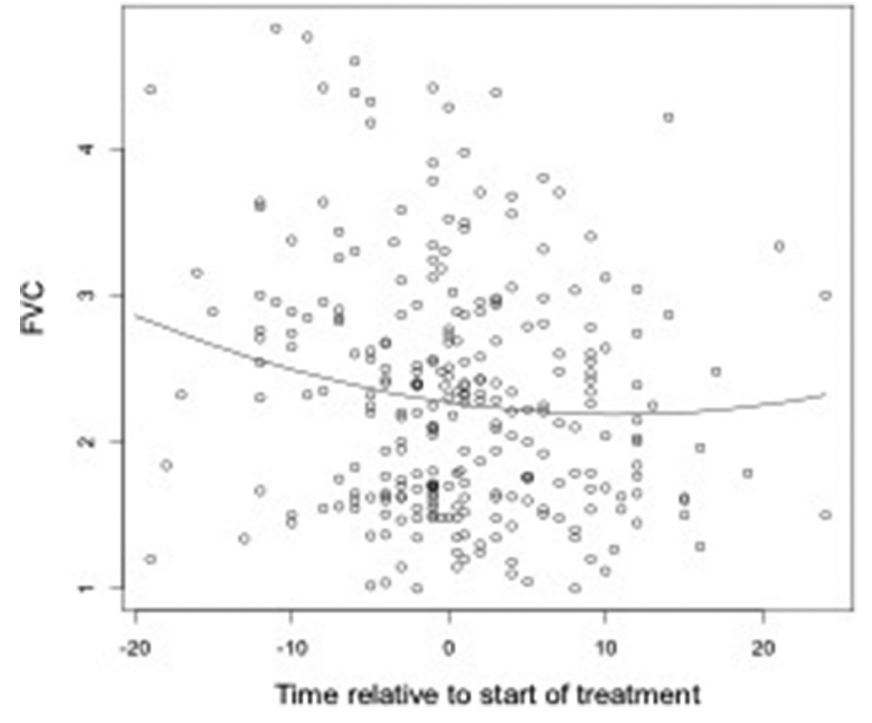

Abstract P30 Figure 1 Showing data plots and average fitted quadratic curve for FVC of patients who received IV Cyclophosphamide therapy. FVC (litres); Time relative to treatment (months)

\section{P31 A RETROSPECTIVE ANALYSIS OF INTERSTITIAL LUNG DISEASE SCREENING IN A REGIONAL CENTRE FOR PATIENTS WITH SCLERODERMA}

${ }^{1} \mathrm{~L}$ Chenciner, ${ }^{2} \mathrm{~F}$ Pearce, ${ }^{2} \mathrm{PC}$ Lanyon, ${ }^{1} \mathrm{SR}$ Johnson. ${ }^{1}$ University of Nottingham, Nottingham, Nottinghamshire; ${ }^{2}$ Nottingham University Hospitals NHS Trust, Nottingham, Nottinghamshire

\subsection{6/thoraxjnl-2015-207770.168}

Background Interstitial Lung Disease (ILD) and Pulmonary Arterial Hypertension (PAH) are the major sources of morbidity and mortality amongst patients with Scleroderma. Specific autoantibodies, anti-Scl70 and anti-centromere (ACA), are associated with ILD and PAH respectively. Screening for ILD and PAH using annual pulmonary function testing (PFT), High Resolution Computed Tomography (HRCT) and Echocardiography respectively, is recommended by the BTS ILD Guidelines, 2008. However, the predictive value of autoantibodies and clinical screening for ILD and PAH, remains unclear in regional centres managing patients with Scleroderma. We hypothesised that an objective scoring system would elucidate lung phenotypes amongst the cohort and confirm original radiology reports for these patients, whilst patients autoantibody profiles would serve a clinical purpose in management. We retrospectively compared identification of ILD by a specialist ILD radiologist, against the use of predictive autoantibody profiling, for the detection of ILD.

Methods 99 patients with Scleroderma, managed in Nottingham, were identified from clinic lists $(n=68)$ or the pathology database, for positive anti-Scl70 and ACA results $(n=31)$. Autoantibody profiles ( $\mathrm{n}=77$ ), including Extractable Nuclear Antigens (ENA) and Myositis Immunoblot, were accessed using the Nottingham University Hospitals Trust pathology database. Existing and accessible HRCT scans $(n=69)$, were evaluated, by a radiologist with a special interest in connective tissue disease-ILD. The Scleroderma Lung Study scoring system was employed, evaluating ground glass opacity, fibrosis, bronchiectasis and honeycombing in three anatomical zones. A binary logistic regression model evaluated the role of autoantibodies in ILD diagnosis. 